\title{
Escher-Trace: a web application for pathway-based visualization of stable isotope tracing data
}

\author{
Avi Kumar ${ }^{1}$, Jack Mitchener ${ }^{1}$, Zachary A. King ${ }^{1}$ and Christian M. Metallo $0^{1,2^{*}}$
}

\author{
* Correspondence: cmetallo@ucsd. \\ edu \\ ${ }^{1}$ Department of Bioengineering, \\ University of California, San Diego, \\ 9500 Gilman Drive, La Jolla, CA \\ 92093, USA \\ ${ }^{2}$ Moores Cancer Center, University \\ of California, San Diego, La Jolla, CA \\ USA
}

\begin{abstract}
Background: Stable isotope tracing has become an invaluable tool for probing the metabolism of biological systems. However, data analysis and visualization from metabolic tracing studies often involve multiple software packages and lack pathway architecture. A deep understanding of the metabolic contexts from such datasets is required for biological interpretation. Currently, there is no single software package that allows researchers to analyze and integrate stable isotope tracing data into annotated or custom-built metabolic networks.

Results: We built a standalone web-based software, Escher-Trace, for analyzing tracing data and communicating results. Escher-Trace allows users to upload baseline corrected mass spectrometer (MS) tracing data and correct for natural isotope abundance, generate publication quality graphs of metabolite labeling, and present data in the context of annotated metabolic pathways. Here we provide a detailed walk-through of how to incorporate and visualize ${ }^{13} \mathrm{C}$ metabolic tracing data into the Escher-Trace platform.

Conclusions: Escher-Trace is an open-source software for analysis and interpretation of stable isotope tracing data and is available at https://escher-trace.github.io/.
\end{abstract}

Keywords: Stable isotope tracing, Metabolism, Escher, Visualization, Web application

\section{Background}

Metabolism plays a central role in all areas of biology. Metabolic reprogramming at the cellular level has been implicated in numerous diseases ranging from diabetes [1, $2]$ to cancer [3, 4]. Understanding metabolic phenotypes involves not only analyzing metabolite abundances (i.e., metabolomics) but also changes in metabolic pathway activity or flux. Stable isotope tracing studies are an effective method for investigating intracellular metabolic fluxes $[5,6]$ that have been increasingly used in the last decade [7]. Insight into pathway activity can be gleaned by applying a stable isotope labeled metabolite to a cell or in vivo system and evaluating where the heavy isotopes are transferred. In recent years, tracing studies have been critical for identifying alterations in tricarboxylic acid [8,9], serine [10], branched chain amino acids [11, 12], and

(c) The Author(s). 2020 Open Access This article is licensed under a Creative Commons Attribution 4.0 International License which permits use, sharing, adaptation, distribution and reproduction in any medium or format, as long as you give appropriate credit to the original author(s) and the source, provide a link to the Creative Commons licence, and indicate if changes were made. The images or other third party material in this article are included in the article's Creative Commons licence, unless indicated otherwise in a credit line to the material. If material is not included in the article's Creative Commons licence and your intended use is not permitted by statutory regulation or exceeds the permitted use, you will need to obtain permission directly from the copyright holder. To view a copy of this licence, visit http://creativecommons.org/licenses/by/4.0/. The Creative Commons Public Domain Dedication waiver (http://creativecommons.org/publicdomain/zero/1.0/) applies to the data made available in this article, unless otherwise stated in a credit line to the data. 
$\mathrm{NAD}(\mathrm{P}) \mathrm{H}$ metabolism [13-15] in a variety of cell and organ systems. Stable isotope tracing experiments utilizing a single tracer and nominal resolution GC-MS analytics in particular have become widely adopted due to their reliability, low cost, and the wealth of information they can provide.

Tracing data sets are initially presented in large data tables which contain the labeling patterns (i.e. isotopologue or mass isotopomer distributions) of all measured compounds in each sample. Interpreting such datasets is best accomplished in the context of metabolic networks and each metabolite's location within metabolic pathways. Additionally, proper reporting of data requires extensive plotting of results, which can be normalized and presented in various ways. The discovery process, which is typically iterative, can be extremely time consuming when dealing with many metabolites or samples. As such, a data visualization platform where isotopologue distributions and related data can be presented graphically in the context of metabolic networks would be very beneficial to the research community. Such a platform would contextualize the data and remove tedious intermediary steps allowing researchers to better focus on validating and interpreting the meaning of their results.

Although numerous software packages are available to analyze metabolomics data, few include capabilities for interpreting stable isotope labeling. XCMS [16], OPENMS [17], as well as vendor-specific software packages allow for integration of mass spectrometry data to quantify metabolite abundances but do not provide options for analyzing metabolite labeling. IsoMetlin [18] allows for identification of isotopically labeled compounds from MS and MS/MS spectra, while software packages such as IsoCor [19], ICT [20], ElemCor [21], and IsoCorrectoR [22] allow users to correct isotopic labeling for natural isotope abundance. MAVEN allows for quantitation of highresolution metabolite labeling as well as natural isotope correction but is not designed for widely available GC-MS systems [23]. Metaboanalyst [24] and Omix [25] allow for visualization of metabolomic and fluxomic datasets in the context of a network but do not provide options for processing or overlaying tracing data. As a result, scientists running tracing studies are required to use multiple software packages to correct their data for natural isotope abundance, perform analysis, prepare figures, and communicate their results.

The most effective way to understand data from tracing experiments is to view metabolite labeling patterns, enrichments, and abundances together on top of a metabolic map. Escher-Trace fills this need by allowing users to overlay multiple types of tracing data on top of Escher metabolic network maps. The software has an interactive, user-friendly, graphical user interface, has a low entry level (i.e. is accessible to users with little tracing experience), and is specifically catered toward users running single tracer studies with GC-MS analytics. Escher-Trace enables users to correct for natural isotope abundance, generate publication quality graphs of metabolite labeling, and present data in the context of pathways. Escher is compatible with BiGG Models [26], providing access to a set of metabolic maps, and the Escher platform provides a comprehensive library of metabolites and metabolic reactions that can be used to seamlessly generate new maps. The ability to generate graphs of multiple data types and compatibility with Escher make Escher-Trace a powerful tool for analyzing and visualizing tracing datasets. We walk through a 
specific use case of how one can employ Escher-Trace to analyze a stable isotope traced data set and generate a figure summarizing the primary findings of the experiment.

\section{Implementation}

Escher-Trace is built on top of the Escher [27] interface, a web based metabolic pathway visualization platform, using javascript and the D3.js library. Escher-Trace allows users to upload stable isotope labeled metabolomic data sets into Escher by clicking the "Import Tracing Data" button in the bottom right hand corner of the screen. Data files can be uploaded in CSV format as either baseline corrected (Additional file 1) or both baseline and natural isotope abundance corrected mass spectrometer counts (Additional file 2) or alternatively in JSON format if reloading a prior Escher-Trace workspace. The required format of uploaded data is specified in the user documentation (https://escher-trace.readthedocs.io/). If uploading data that is not corrected for natural isotope abundance, the user will be asked to indicate which type of tracer was used in their data set (e.g. $13 \mathrm{C}, 15 \mathrm{~N}, 2 \mathrm{H}$ ). When uploading data for the first time, the user will be instructed to organize their samples into groups based on experimental conditions. Sample data within the same group will be averaged together and presented with standard deviation in graphs. Data sets with over one hundred samples organized into over forty groups have successfully been uploaded to the tool. However, the largest default color scheme in Escher-Trace contains fifteen color entries, if more groups are included, the user can create and utilize larger color schemes by selecting Graph Attribute $\rightarrow$ Color Scheme from the Escher-Trace menu, additional instruction can be found in the user documentation. After organizing sample data files into groups, isotopologue distributions which correspond to metabolites included in the Escher map will be displayed next to the corresponding Escher metabolite node. Data is mapped by connecting the BiGG IDs of metabolites entered by the user to the corresponding Escher nodes. BiGG IDs are standardized identifiers of metabolites included in the BiGG Models database [26], which allows for the connection of Escher map nodes to genome scale metabolic models and external databases such as KEGG [28] and BioCyc [29]. All unmapped metabolite data is displayed on the left-hand side of the map. Data that is uncorrected for natural isotope abundance is corrected using the user selected tracer type, metabolite formula, actual measurements, natural stable isotope enrichment information, and the algorithm presented by Fernandez et al. [30]. Matrix calculations are performed using functions from the math.js library. The correction algorithm used by Escher-Trace is best suited to correct small molecule metabolite data generated by a nominal resolution mass spectrometer. Data which does not fit these conditions can still be visualized in EscherTrace, but must be corrected first using a separate correction software and uploaded as a corrected csv data file (the format for which can be found in the user documentation). A comparison of results obtained from the correction algorithms of Escher-Trace and IsoCor can be found in Additional file 3. Escher-Trace can visualize metabolite isotope labeling, enrichment, and abundance as single bar or stacked bar plots for steady state labeling studies or as line graphs for time- 
course/kinetic studies (Fig. 1). Isotopologue distributions are calculated by dividing the abundance of each isotopologue by the sum total of all isotopologues of the metabolite.

The user can interact with their data by two means (1) using the Escher-Trace Menu located on the right-hand side of the screen or (2) right-clicking individual graphs. With the Escher-Trace menu, the user can make changes which affect graphs across the network such as altering data types or metabolites that are visualized, normalizing abundances of metabolites, and saving the Escher-Trace workspace in JSON format. By right-clicking individual graphs, the user can download the selected graph in SVG or PNG format, remove the graph from the map, view the data used to generate the graph, or generate additional graphs relating to the specific metabolite including data for additional fragments.

\section{Results and discussion}

To demonstrate a typical use case of Escher-Trace, we will analyze data from the Huh7 hepatocellular carcinoma cell line grown with $\left[\mathrm{U}_{-}{ }^{13} \mathrm{C}_{5}\right.$ ]glutamine in normoxic $(21 \%$ oxygen) and hypoxic (1\% oxygen) conditions. Hypoxia induces a classical metabolic reprogramming phenotype in highly proliferative cells [31]. We will walk through how a user might identify reprogramming induced by hypoxia and generate a figure that communicates this phenotype with Escher-Trace.

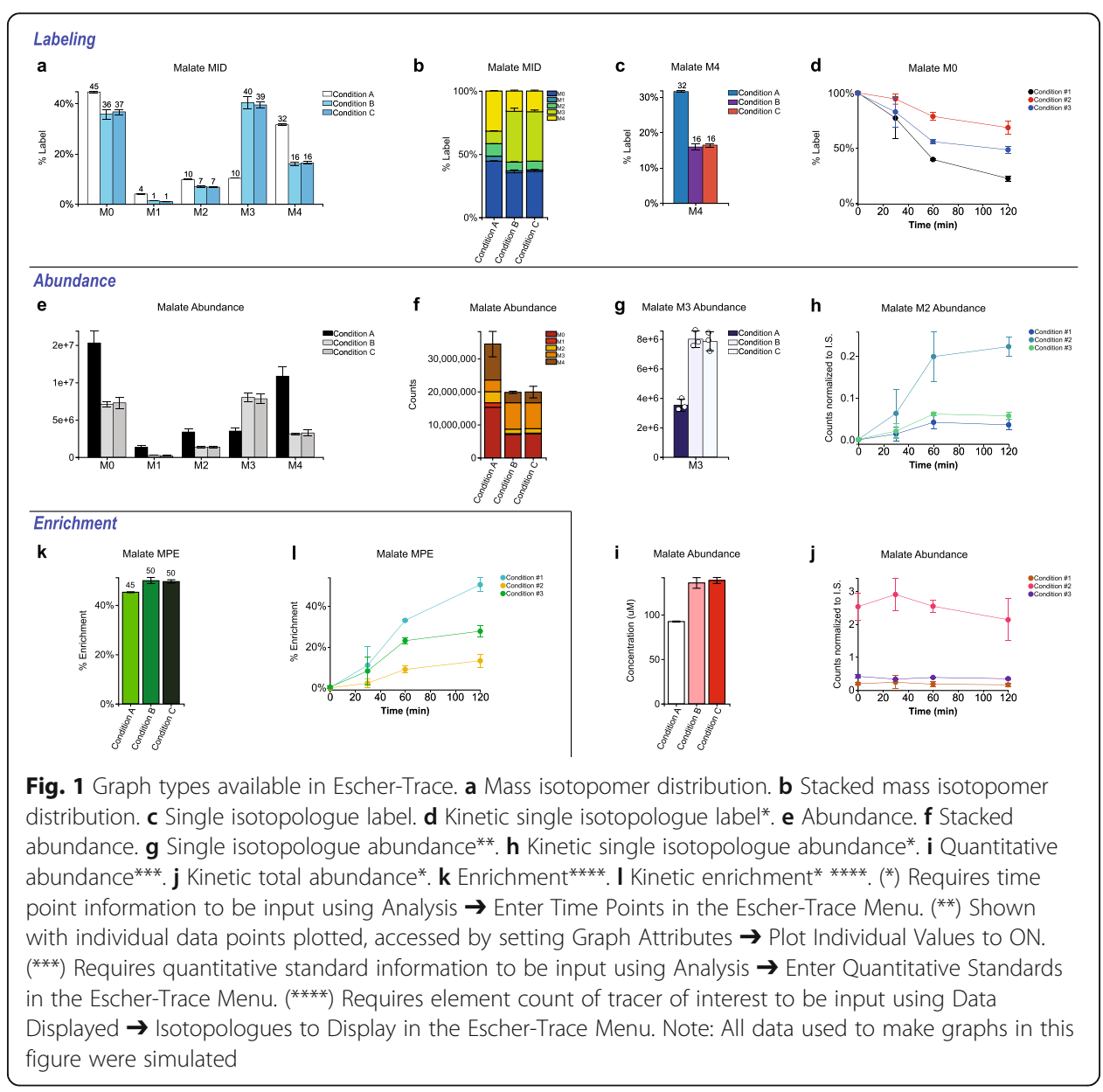


First, we must upload our raw mass spectrometer counts CSV file (available as Additional file 1) which were analyzed via nominal resolution gas chromatography-mass spectrometry (GC-MS) and integrated with an available MS integration software package. The data is baseline corrected but remains uncorrected for natural isotope abundance, so we upload the CSV file to Escher-Trace as an uncorrected CSV file and select the tracer type as " ${ }^{13} \mathrm{C}$. We organize the sample data files into two distinct groups which are named "Normoxia" and "Hypoxia" to represent each experimental condition. After submitting the sample order, isotopologue distributions of our data appear on top of the Escher map (Fig. 2). At first glance, one can identify that our experimental conditions produce distinct labeling patterns in TCA cycle intermediates.

Upon closer observation of the isotopologue distributions for TCA intermediates, we can identify a unique labeling distribution on citrate. Specifically, we observe increased relative abundance of M5 citrate label (over 6x increased) in hypoxia compared to normoxia (Fig. 3a). The number of isotopologues visualized in Escher-Trace graphs can be adjusted for clarity by selecting Data Displayed $\rightarrow$ Isotopologues to Display from the Escher-Trace menu and reducing the isotopologue limit for the metabolite of interest. Using the metabolic map, we can identify that M5 citrate can only be generated reductively (in the counter-clockwise direction) from alpha-ketoglutarate (aKG). If aKG is oxidized in the clockwise direction of the TCA cycle, M4 isotopologues of the remaining TCA intermediates will form. This includes M4 citrate which is generated from M4 oxaloacetate and unlabeled mitochondrial acetyl-CoA derived predominantly from unlabeled glucose-derived pyruvate. Increased M5 citrate formation is now known

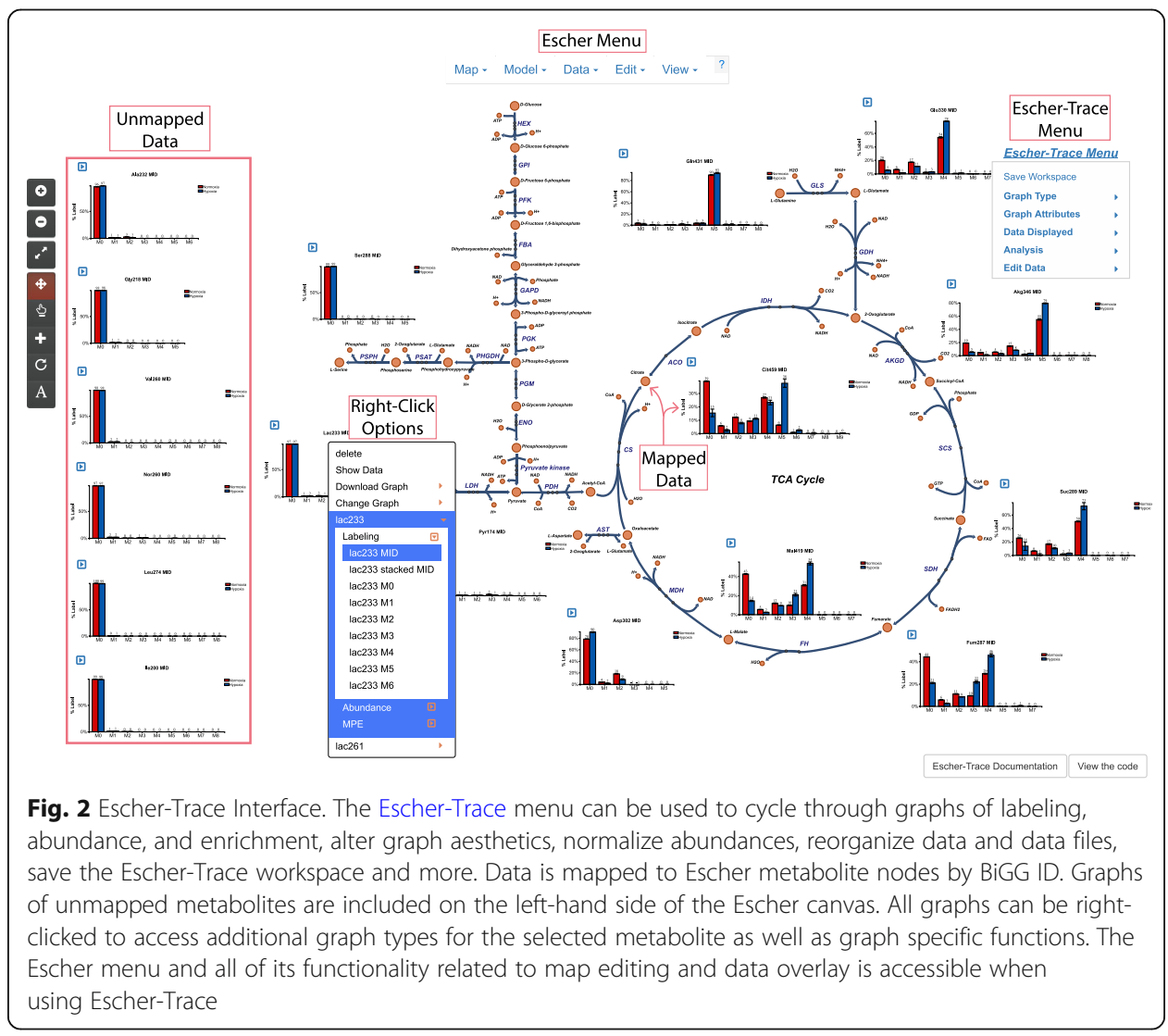




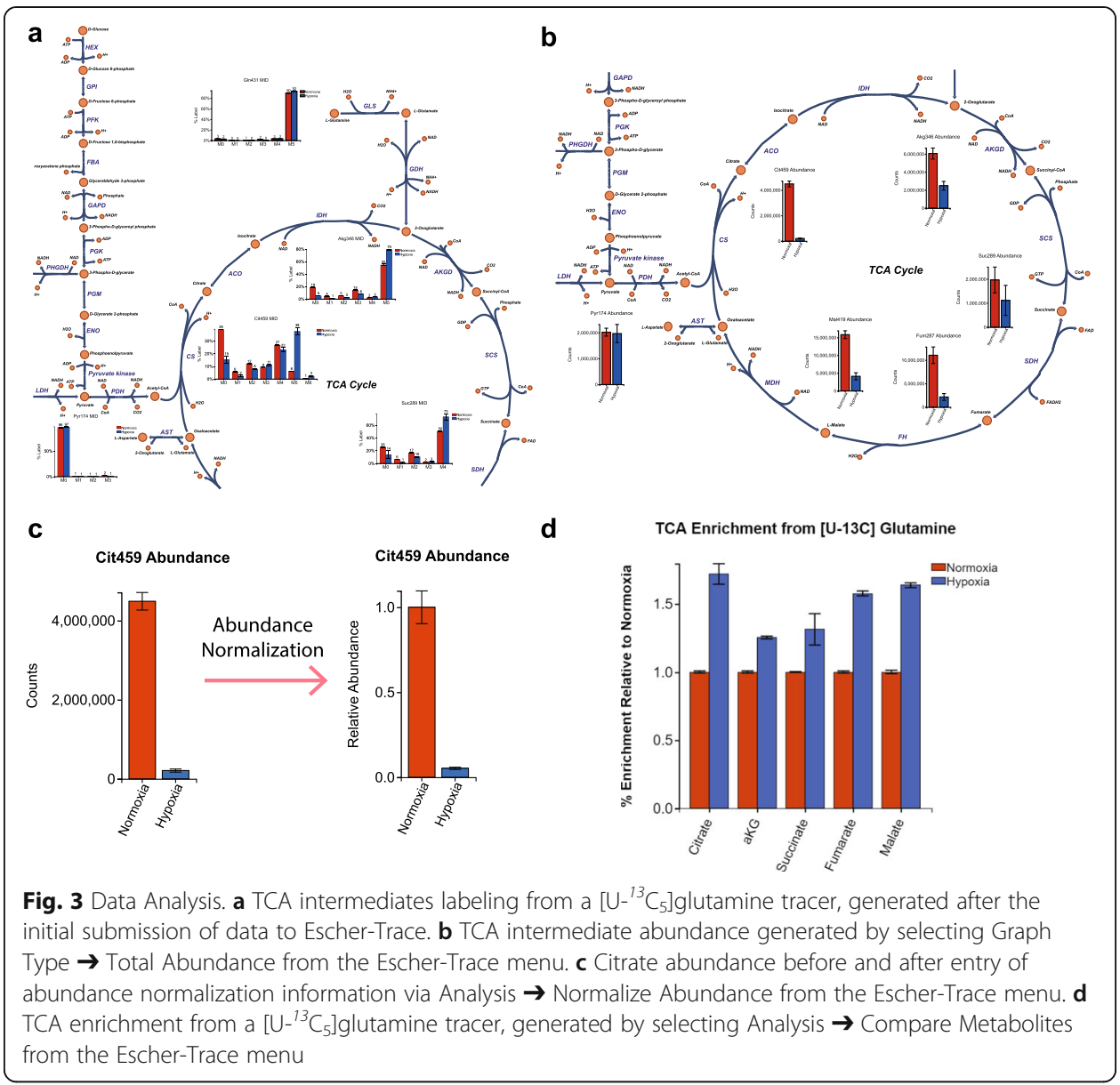

to be demonstrative of upregulated reductive carboxylation flux which has been observed in highly proliferative cells cultured in hypoxia [32].

To get a broader overview of how metabolite abundances are altered between the experimental conditions we select on the Escher-Trace menu Graph Type $\rightarrow$ Total Abundance. This causes all metabolite data to switch from isotopologue distributions to abundances (Fig. 3b). We see that there appears to be a distinct decrease in the raw counts of TCA intermediates in cells under hypoxia. However, to properly analyze how intracellular abundances are impacted by hypoxia, we need to normalize our data based on our experimental workflow. Metabolite abundance normalization is performed by selecting Analysis $\rightarrow$ Normalize Abundances on the Escher-Trace menu. Here a norvaline internal standard was spiked into all samples, so we select nor260, one of the two norvaline fragments we detect with our GC-MS method, for normalization of all metabolites. Additionally, cell count data can be entered to obtain per cell quantitation of metabolites (normoxia: 638333 cells, hypoxia: 668750 cells). We can also optionally select an experimental condition to normalize our data to simplify data presentation; in our case we present data relative to normoxia. After submitting our selections, total abundance graphs are rescaled (Fig. 3c). One can see that the per cell abundances of TCA intermediates are decreased in hypoxic conditions, as previously noted [32].

We can streamline data communication by producing graphs of data across metabolites. These types of graphs can by generated in Escher-Trace by selecting Analysis $\rightarrow$ 
Compare Metabolites in the Escher-Trace menu. This option allows the user to select metabolites, experimental conditions, and data types (metabolite abundance, enrichment, or individual isotopologue labeling) to include in a bar graph. Using this feature, we generated a summary figure highlighting increased TCA intermediate enrichment from $\left[\mathrm{U}_{-}{ }^{13} \mathrm{C}_{5}\right]$ glutamine in hypoxia (Fig. $3 \mathrm{~d}$ ).

Finally, to simplify the data presented on the metabolic map, we can select to only display data for metabolites of interest, glycolytic and TCA intermediates in this case, by using Data Displayed $\rightarrow$ Metabolites to Display in the Escher-Trace menu. Figures of additional data types, including individual isotopologue labeling, metabolite abundance, and mole percent enrichment, as well as additional plot types (single or stacked bar plots) can be accessed using the context menu, by right-clicking any Escher-Trace graph, selecting a metabolite fragment, and then selecting the data and plot type. The title and $y$-axis labels of all graphs can be edited by clicking on them and entering in new text as needed for clarity. Finally, labeling diagrams can be introduced using Data Displayed $\rightarrow$ Create Carbon Diagram, to generate the final figure (Fig. 4) This publication-quality figure can be downloaded as an SVG or PNG file using the Escher menu selecting Map $\rightarrow$ Export as SVG or PNG. Individual graphs can be independently downloaded by right-clicking them and selecting Download $\rightarrow$ SVG or PNG. The Escher-Trace workspace itself can be downloaded by selecting Save Workspace

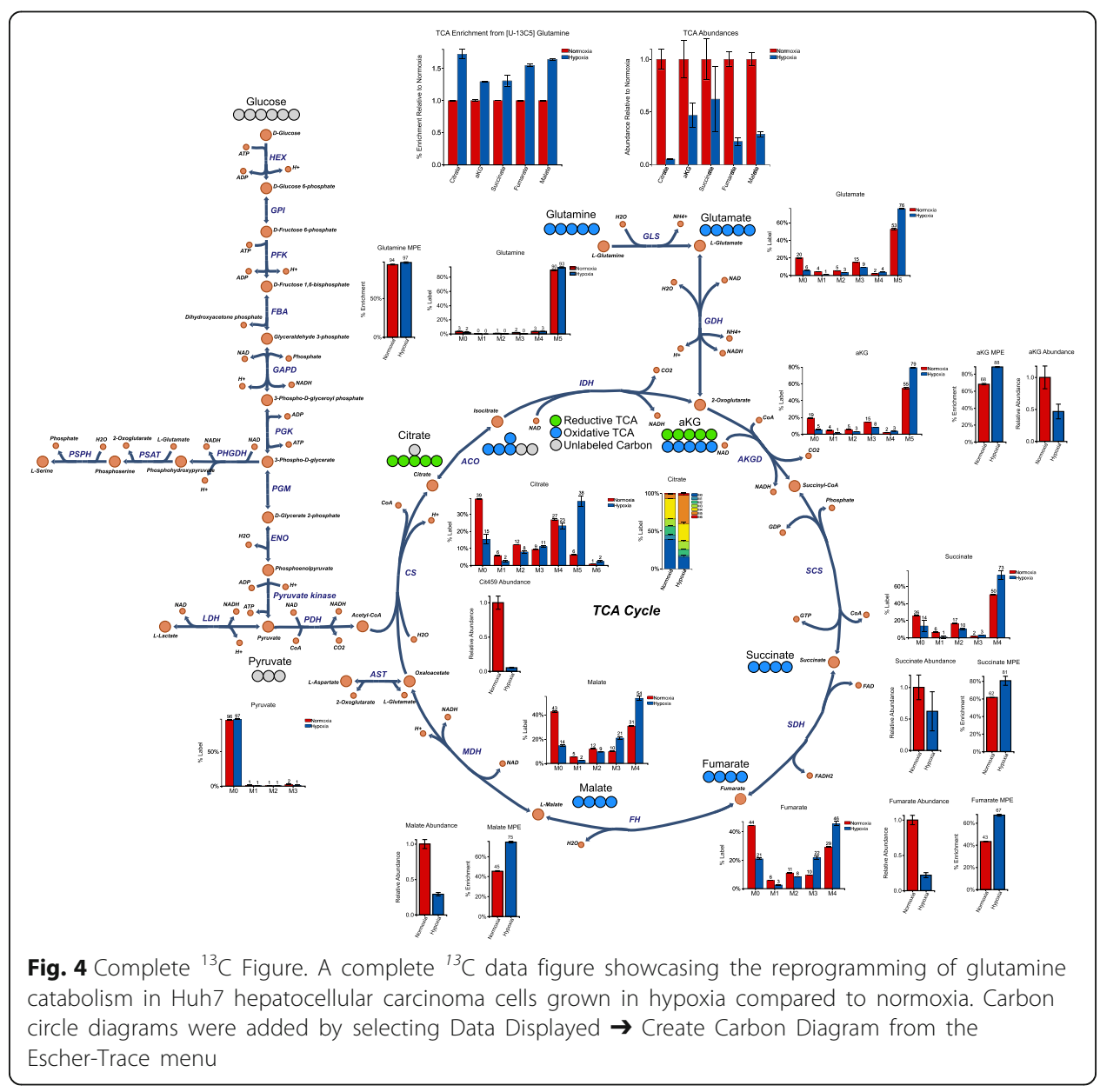


from the Escher-Trace menu. This workspace file can be sent to collaborators and reloaded in Escher-Trace to communicate findings or perform further analyses.

\section{Conclusions}

Escher-Trace is a web-based software for visualizing and interpreting stable isotope tracing data in the context of a metabolic network. We have shown that Escher-Trace can facilitate calculation and presentation of labeling patterns resulting from alterations in pathway activity (i.e. hypoxia) and overlay results on an Escher pathway map. By providing a tool which allows users to correct for natural isotope abundance, visualize labeling patterns, and generate scientific figures all through a simple graphical user interface, we believe Escher-Trace is a significant addition to the software available to metabolic researchers.

\section{Availability and requirements}

- Project name: Escher-Trace.

- Project home page: http://escher-trace.github.io

- Operating system(s): Platform independent.

- Programming language: HTML and Javascript.

- License: MIT License.

- Any restrictions to use by non-academics: none.

\section{Supplementary information}

Supplementary information accompanies this paper at https://doi.org/10.1186/s12859-020-03632-0.

Additional file 1. Escher-Trace Uncorrected Example Data File.csv This file contains the data set used to generate all graphs in the manuscript. Data was generated by culturing Huh7 hepatoma cells, provided by M. Hemann, in normoxia or hypoxic (1\% oxygen) conditions for $48 \mathrm{~h}$ with Dulbecco's Modified Eagle Media containing $4 \mathrm{mM}\left[\mathrm{U}^{13}{ }^{13} \mathrm{C}_{5}\right.$ ]glutamine. This file can be uploaded to Escher-Trace as described in the Implementation section. The data set is also available at https://escher-trace.readthedocs.io/.

Additional file 2. Escher-Trace Corrected Example Data File.csv This file contains the same data set as Additional file 1, however the data has been pre-corrected for natural isotope abundance. This file is also available in the Escher-Trace documentation (https://escher-trace.readthedocs.io/) where a walkthrough of how the file needs to be constructed and uploaded to Escher-Trace can be found.

Additional file 3. Comparison of Escher-Trace and IsoCor NAC.docx This file contains a comparison of stable isotope correction results obtained from Escher-Trace and IsoCor.

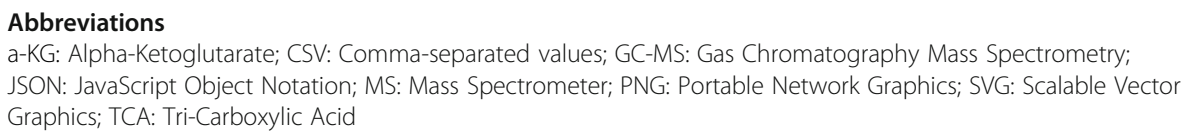
Novo Nordisk Foundation through the Center for Biosustainability at the Technical University of Denmark \#NNF10CC1016517 (ZAK). The funding bodies did not play any roles in the design of the study and collection, analysis, and interpretation of data and in writing the manuscript. 


\section{Availability of data and materials}

All data generated or analyzed during this study are included in this published article [and its supplementary information files]. These files can also be accessed on the Escher-Trace version 1.1 site at https://www.escher-trace. github.io/. Escher-Trace documentation, which has in-depth walkthroughs of all Escher-Trace functionality, can be found at https://escher-trace.readthedocs.io/. The source code of Escher-Trace can be found at https://github.com/ escher-trace/escher-trace.github.io.

\section{Ethics approval and consent to participate}

Not applicable.

\section{Consent for publication}

Not applicable.

\section{Competing interests}

The authors declare that they have no conflict of interest with the contents of this article.

Received: 17 February 2020 Accepted: 23 June 2020

Published online: 10 July 2020

\section{References}

1. Wang TJ, Larson MG, Vasan RS, Cheng S, Rhee EP, Mccabe E, et al. Metabolite profiles and the risk of developing diabetes. Nat Med. 2011;17:448-54. https://doi.org/10.1038/nm.2307.

2. Wellen KE, Hotamisligil GS. Inflammation, stress, and diabetes. J Clin Invest. 2005;115:1111-9. https://doi.org/10.1172/ JCI25102.

3. Deberardinis RJ, Lum JJ, Hatzivassiliou G, Thompson CB. The biology of cancer : metabolic reprogramming fuels cell growth and proliferation. Cell Metab. 2008;7:11-20. https://doi.org/10.1016/j.cmet.2007.10.002.

4. Hsu PP, Sabatini DM. Cancer cell metabolism: Warburg and beyond. Cell. 2008;134:703-7. https://doi.org/10.1016/j.cell. 2008.08.021.

5. Sauer U. Metabolic networks in motion: 13C-based flux analysis. Mol Syst Biol. 2006;2:62. https:/doi.org/10.1038/ msb4100109.

6. Wiechert W. 13C metabolic flux analysis. Metab Eng. 2001;3:195-206. https://doi.org/10.1006/mben.2001.0187.

7. Jang C, Chen L, Rabinowitz JD. Metabolomics and isotope tracing. Cell. 2018;173:822-37. https://doi.org/10.1016/j.cell. 2018.03.055.

8. Fendt S, Bell EL, Keibler MA, Olenchock BA, Mayers JR, Wasylenko TM, et al. Reductive glutamine metabolism is a function of the a-ketoglutarate to citrate ratio in cells. Nat Commun. 2013;4:1-11. https://doi.org/10.1038/ncomms3236.

9. Cordes T, Metallo CM. Quantifying intermediary metabolism and lipogenesis in cultured mammalian cells using stable isotope tracing and mass spectrometry. In: D'Alessandro A, editor. High-throughput metabolomics: methods and protocols. New York: Springer New York; 2019. p. 219-41. https://doi.org/10.1007/978-1-4939-9236-2_14.

10. Locasale JW, Grassian AR, Melman T, Lyssiotis CA, Mattaini KR, Bass AJ, et al. Phosphoglycerate dehydrogenase diverts glycolytic flux and contributes to oncogenesis. Nat Publ Gr. 2011;43. https://doi.org/10.1038/ng.890.

11. Mayers JR, Torrence ME, Danai LV, Papagiannakopoulos T, Davidson SM, Bauer MR, et al. Tissue of origin dictates branched-chain amino acid metabolism in mutant Kras-driven cancers. Science. 2016;353:1161-5. https://doi.org/10. 1126/science.aaf5171.

12. Wallace M, Green CR, Roberts LS, Lee YM, McCarville JL, Sanchez-Gurmaches J, et al. Enzyme promiscuity drives branched-chain fatty acid synthesis in adipose tissues. Nat Chem Biol. 2018;14:1021-31. https://doi.org/10.1038/s41589018-0132-2.

13. Badur MG, Muthusamy T, Parker SJ, Ma S, Mcbrayer K, Cordes T, et al. Oncogenic R132 IDH1 mutations limit NADPH for De novo lipogenesis through (D)2-Hydroxyglutarate production in Fibrosarcoma cells. Cell Rep. 2018;25:1018-26. https://doi.org/10.1016/j.celrep.2018.09.074.

14. Fan J, Ye J, Kamphorst JJ, Shlomi T, Thompson CB, Rabinowitz JD. Quantitative flux analysis reveals folate-dependent. Nature. 2014;510:298-302. https://doi.org/10.1038/nature13236.

15. Lewis CA, Parker SJ, Fiske BP, Mccloskey D, Gui DY, Green CR, et al. Tracing compartmentalized NADPH metabolism in the cytosol and mitochondria of mammalian cells. Mol Cell. 2014;55:1-11. https://doi.org/10.1016/j.molcel.2014.05.008.

16. Tautenhahn R, Patti G, Rinehard D, Siuzdak G. XCMS online: a web-based platform to process untargeted metabolomic data. Anal Chem. 2013;84:5035-9. https://doi.org/10.1021/ac300698c.XCMS

17. Röst HL, Sachsenberg T, Aiche S, Bielow C, Weisser H, Aicheler F, et al. OpenMS : a flexible open-source software platform for mass spectrometry data analysis. Nat Methods. 2016;13:741-8. https://doi.org/10.1038/nmeth.3959.

18. Cho K, Mahieu N, Ivanisevic J, Uritboonthai W, Chen YJ, Siuzdak G, et al. IsoMETLIN: a database for isotope-based metabolomics. Anal Chem. 2014;86:9358-61. https://doi.org/10.1021/ac5029177.

19. Millard $P$, Delépine $B$, Guionnet $M$, Heuillet $M$, Bellvert F, Létisse F, et al. IsoCor: isotope correction for high-resolution MS labeling experiments. Bioinformatics. 2019;35:4484-7. https://doi.org/10.1093/bioinformatics/btz209.

20. Jungreuthmayer C, Neubauer S, Mairinger T, Zanghellini J, Hann S. ICT: isotope correction toolbox. Bioinformatics. 2016; 32:154-6. https://doi.org/10.1093/bioinformatics/btv514.

21. Du D, Tan L, Wang Y, Peng B, Weinstein JN, Wondisford FE, et al. ElemCor: accurate data analysis and enrichment calculation for high-resolution LC-MS stable isotope labeling experiments. BMC Bioinformatics. 2019;20:1-9. https://doi. org/10.1186/s12859-019-2669-9.

22. Heinrich P, Kohler C, Ellmann L, Kuerner P, Spang R, Oefner PJ, et al. Correcting for natural isotope abundance and tracer impurity in MS-, MS/MS- and high-resolution-multiple-tracer-data from stable isotope labeling experiments with IsoCorrectoR. Sci Rep. 2018;8:1-10. https://doi.org/10.1038/s41598-018-36293-4.

23. Melamud E, Vastag L, Rabinowitz JD. Metabolomic analysis and visualization engine for LC-MS data. Anal Chem. 2010; 82:9818-26. https://doi.org/10.1016/j.physbeh.2017.03.040. 
24. Chong J, Soufan O, Li C, Caraus I, Li S, Bourque G, et al. MetaboAnalyst 4.0: towards more transparent and integrative metabolomics analysis. Nucleic Acids Res. 2018;46:486-94. https://doi.org/10.1093/nar/gky310.

25. Droste P, Miebach S, Niedenführ S, Wiechert W, Nöh K. Visualizing multi-omics data in metabolic networks with the software Omix-a case study. BioSystems. 2011;105:154-61. https://doi.org/10.1016/j.biosystems.2011.04.003.

26. Norsigian CJ, Pusarla N, McConn JL, Yurkovich JT, Dräger A, Palsson BO, et al. BiGG models 2020: multi-strain genomescale models and expansion across the phylogenetic tree. Nucleic Acids Res. 2019;48:1-5. https://doi.org/10.1093/nar/ gkz1054.

27. King ZA, Dräger A, Ebrahim A, Sonnenschein N, Lewis NE, Palsson BO. Escher: a web application for building, sharing, and embedding data-rich visualizations of biological pathways. PLoS Comput Biol. 2015;11:1-13. https://doi.org/10.1371/ journal.pcbi.1004321.

28. Qi M, Wang R, Jing B, Jian F, Ning C, Zhang L. KEGG: Kyoto encyclopedia of genes and genomes. Nucleic Acids Res. 2000;28:27-30. https://doi.org/10.1016/j.meegid.2016.07.022.

29. Karp PD, Billington R, Caspi R, Fulcher CA, Latendresse M, Kothari A, et al. The BioCyc collection of microbial genomes and metabolic pathways. Brief Bioinform. 2018;20:1085-93. https://doi.org/10.1093/bib/bbx085.

30. Fernandez CA, Des Rosiers C, Previs SF, David F, Brunengraber H. Correction of $13 \mathrm{C}$ mass isotopomer distributions for natural stable isotope abundance. J Mass Spectrom. 1996;31:255-62. https://doi.org/10.1002/(SICI)1096-9888(199603)31: 3<255::AID-JMS290>3.0.CO;2-3.

31. Semenza GL. HIF-1 : upstream and downstream of cancer metabolism. Curr Opin Genet Dev. 2010;20:1-10. https://doi. org/10.1016/.gde.2009.10.009.HIF-1.

32. Metallo CM, Gameiro PA, Bell EL, Mattaini KR, Yang J, Hiller K, et al. Reductive glutamine metabolism by IDH1 mediates lipogenesis under hypoxia. Nature. 2012;481:380-4. https://doi.org/10.1038/nature10602.

\section{Publisher's Note}

Springer Nature remains neutral with regard to jurisdictional claims in published maps and institutional affiliations.

Ready to submit your research? Choose BMC and benefit from:

- fast, convenient online submission

- thorough peer review by experienced researchers in your field

- rapid publication on acceptance

- support for research data, including large and complex data types

- gold Open Access which fosters wider collaboration and increased citations

- maximum visibility for your research: over $100 \mathrm{M}$ website views per year

At $B M C$, research is always in progress.

Learn more biomedcentral.com/submissions 